\title{
Apoptotic Vascular Smooth Muscle Cell Depletion via BCL2 Family of Proteins in Human Ascending Aortic Aneurysm and Dissection
} \author{
\& Ahmet Ruchan Akar ${ }^{1,2}$ \\ 1 Department of Cardiovascular Surgery, Heart Center, Ankara University School of Medicine, Ankara, Turkey \\ 2 Ankara University Stem Cell Institute, Ankara, Turkey \\ 3 Ankara University Biotechnology Institute, Ankara, Turkey \\ 4 Department of Histology and Embryology, Ankara University, Ankara, Turkey \\ 5 Department of Molecular Biology and Genetics, Bilkent University, Ankara, Turkey
}

Serkan Durdu, ${ }^{1,2,3}$ Gunseli C. Deniz, ${ }^{2,3}$ Deniz Balci, ${ }^{3,4}$ Cagin Zaim, ${ }^{1}$ Arin Dogan, ${ }^{3}$ Alp Can, ${ }^{2,4}$ Kamil C. Akcali ${ }^{5}$

\section{Keywords}

Aortic aneurysm; Apoptosis; BCL2

homologous antagonist-killer protein;

Dissecting aneurysm; Smooth muscle cells.

\section{Correspondence}

A. R. Akar, M.D., FRCS CTh., Department of Cardiovascular Surgery, Heart Center, University of Ankara School of Medicine, Dikimevi, Ankara 06340, Turkey.

Tel.: +90 (533) 6460684;

Fax: +90 (312) 3625639;

and

Ankara University Stem Cell Institute, Cevizlidere, Balgat, Ankara 06520, Turkey.

Tel.: +90 (312) 5836660;

Fax: +90 (312) 5836605;

E-mail: akarruchan@gmail.com or

akar@ankara.edu.tr

doi: $10.1111 / 1755-5922.12007$

\begin{abstract}
SUMMARY
Aims: This study investigates the expression patterns of BCL2 (B-cell CLL/lymphoma2) family of proteins and the extent of vascular smooth muscle cell (VSMC) apoptosis in thoracic aortic aneurysms (TAA), type-A aortic dissections (TAD), and nondilated ascending aortic samples. Methods: Aortic wall specimens were obtained from patients undergoing surgical repair for TAA $(n=24)$, TAD $(n=20)$, and normal aortic tissues from organ donors $(\mathrm{n}=6)$. The expression pattern of BCL2, BCL2L1 (BCL2-like1), BAKl (BCL2-antagonist/ killer1), and BAX (BCL2-associated X protein) proteins was investigated by immunohistochemistry. Furthermore, colocalization of alpha smooth muscle actin (ACTA2) and caspase 3 (CASP3) in aortic VSMCs was analyzed by double-immunofluorescence staining. Onset of DNA fragmentation was measured by TUNEL assay. Results: Apoptotic index was significantly increased in both TAD group $(31.3 \pm 17.2, P<0.001)$ and TAA group $(21.1 \pm 12.7$, $P=0.001)$ relative to control aortas $(2.0 \pm 1.2)$. Anti-CASP3 and ACTA2 double-immunostaining confirmed apoptosis in VSMCs in TAA and TAD groups but not in controls. Proapoptotic BAX expression was significantly elevated in VSMCs of TAA patients, compared with that of controls $(\mathrm{OR}=20 ; P=0.02 ; 95 \% \mathrm{CI}, 16-250)$. In contrast, antiapoptotic BCL2L1 expression was higher in controls compared with that of TAA group (OR = 11.2; $P=0.049 ; 95 \%$ CI, 1.0-123.9). Furthermore, BAX/BCL2 ratio was significantly increased in both TAA $(1.2 \pm 0.7, P<0.001)$ and TAD $(0.6 \pm 0.4, P=0.05)$ groups relative to controls $(0.2 \pm 0.1, P<0.001)$. Conclusions: Apoptotic VSMC depletion in human TAA/TAD is associated with disturbance of the balance between proapoptotic and antiapoptotic members of the BCL2 family proteins, which may have a role in the pathogenesis of vascular remodelling in aortic disease. In light of the future studies, targeting apoptotic pathways in TAA and TAD pathogenesis may provide therapeutic benefits to patients by slowing down the progression and even possibly preventing the TAD.
\end{abstract}

\section{Introduction}

Apoptosis, as introduced by Kerr et al. [1], is a process of noninflammatory cell death with distinctive morphological and biochemical features. It is crucial for normal development of several systems including the cardiovascular system. Vascular smooth muscle cell (VSMC) death by apoptosis has increasingly been recognized in normal physiological circumstances such as closure of ductus arteriosus and in several human vascular pathologies [2, 3]. It has been demonstrated that medial VSMC density decreases in human abdominal aortic aneurysm (AAA) tissues associated with evidence of chronic, low-level VSMC death, fragmentation of elastin, and matrix degradation [4-6]. However, neuroectodermal origin of VSMCs located in ascending aorta differs from abdominal aortic counterparts. VSMC depletion may also be involved in ascending aortic aneurysm formation, initiation of dissection, propagation, and rupture. Indeed, He et al. [7] had previously documented increased amount of apoptotic VSMCs by terminal transferase deoxyuridine triphosphate nick-end labeling (TUNEL) in the media of both thoracic aortic aneurysms (TAA) and Stanford type-A dissections (TAD).

Extensive studies regarding the molecular mechanisms of apoptosis made it clear that proteins belonging to the BCL2 (B-cell CLL/lymphoma 2) family have a critical role in regulating mitochondrion-mediated apoptosis. BCL2 family of proteins consists of both proapoptotic and antiapoptotic members that are involved in the regulation of apoptosis by forming homo/heterodimers on the outer mitochondrion membrane [8]. The relative concentrations 
of these proteins determine the survival or death of a cell by controlling the release of mitochondrial apoptogenic factors, cytochrome c, and apoptosis-inducing factors, which activate the downstream phases, including the activation of the caspases [9]. The ratio of antiapoptotic BCL2 family proteins (BCL2 and BCL2L1 [BCL2-like 1]) to proapoptotic members (BAX [BCL2associated $\mathrm{X}$ protein], BID [BH3 interacting domain death agonist], BIK [BCL2-interacting killer; apoptosis-inducing], and BAK1 [BCL2-antagonist/killer 1]) has been shown to have a critical role in whether apoptotic cell death is initiated or the cell continues to survive [10].

The objective of this study was to elucidate the role of BCL2 family of proteins in aortic VSMC apoptosis in TAD and TAA patients. Thus, we investigated the relevance of apoptosis together with the expression patterns of the different BCL2 family of proteins and caspase 3 (CASP3, apoptosis-related cysteine peptidase) using immunolabeling in successive sections of aorta from TAD/ TAA patients and controls. Our results may provide useful insights into the differential involvement of the BCL2 family of proteins and CASP3 in human TAA and TAD.

\section{Methods}

\section{Aneurysmal, Dissected, and Normal Aortic Tissues}

The Research Ethics Committee of the Ankara University School of Medicine approved the study protocol on February 19, 2007 (107-2808), and all subjects gave written informed consent to participate in the study. Between May 2007 and March 2011, fullthickness ascending aortic wall specimens, which were collected from greater curvature of distal aortic root neighboring to anterolateral portion of sinotubular junction, were obtained from patients undergoing surgical repair of TAA $(n=24)$, or TAD $(\mathrm{n}=20)$, and compared with normal aortic tissues from organ donors $(n=6)$. Type-A aortic dissection (TAD) was defined as any dissection that involved the ascending aorta and/or aortic arch. Particular care was taken to obtain both layers of the dissected aortic wall in the TAD group. The baseline characteristics of the patients participating in the study are summarized in Table 1. Patients were not enrolled in the study if any one of the following exclusion criteria were met: (1) the diagnosis of Marfan syndrome (based on the revised criteria of De Paepe [11]), Ehlers-Danlos syndrome, Loeys-Dietz syndrome, aneurysm-osteoarthritis syndrome, and familial TAA and/or dissections (caused by mutations in the alpha smooth muscle actin [ACTA2] or MYH1l gene); (2) presence of unicuspid or bicuspid aortic valve; (3) aortitis in any form or evidence for endocarditis; (4) history of cocaine abuse; and (5) iatrogenic aortic dissection or any TAD that does not involve the ascending aorta $10 \mathrm{~mm}$ above the sinotubular junction. Tissue samples collected at the time of operation were placed immediately into $10 \%$ neutral buffered formalin solutions, processed, and embedded in paraffin. Thickness measurements of tunica media and adventitia were performed in ACTA2-stained coverslips using LSM 510 software (Carl Zeiss, Jena, Germany) coupled to an Axiovert $100 \mathrm{M}$ microscope (Carl Zeiss) with a $10 \times$ objective. The measurements considered for each vessel were the mean values of the three measurements.

\section{Immunohistochemistry}

Five-micrometer deparaffinized sections were incubated for $30 \mathrm{~min}$ in $0.3 \%$ hydrogen peroxide in methanol to quench endogenous peroxidase activity. Slides were washed in phosphate buffered saline (PBS) for $10 \mathrm{~min}$, three times, and incubated with blocking serum (normal goat serum 1.5\%, bovine serum albumin $2 \%$, Triton X-100 0.1\%) for one hour at room temperature. Primary antibodies against BCL2, BCL2L1, BAX, BAKl $(1 \mu \mathrm{g} / \mathrm{mL}$; Santa Cruz Biotechnology, Inc., Santa Cruz, CA, USA; Catalog numbers; BAX: sc-526 [rabbit]; BAK1: sc-832 [rabbit]; BCL2: sc492 [rabbit]; BCL2L1: sc-8392 [mouse]) were applied in successive paraffin sections and kept at $4{ }^{\circ} \mathrm{C}$ for overnight, followed by streptavidin-HRP (DakoCytomation, Glostrup, Denmark; P0397) for $15 \mathrm{~min}$ at room temperature. After $10 \mathrm{~min}$ of PBS wash, color developments were achieved by incubation with Liquid DAB+ (DakoCytomation). The slides were then counterstained with hematoxylin (HEE) and mounted using Faramount Aqueous Mounting Medium (DakoCytomation). For negative controls, we omitted primary antibodies. To analyze immunoreactivity, the number of immunopositive cells in sections was semiquantitatively scored [12]. The scoring is as follows: 0 , not present; 1 , light; 2, moderate; 3 , high.

\section{In Situ End-Labeling of DNA Fragments (TUNEL)}

DNA fragmentation was detected in situ with a DNTT (terminal deoxynucleotidyl transferase)-mediated fluorescein-dUTP labeling kit (Roche Diagnostics, Mannheim, Germany) in the parallel sections according to manufacturer's instructions. To detect all nuclei, we used 7-aminoactinomycin D (7-AAD; Sigma, St Louis, MO, USA). For each aortic sample, at least 10 grid fields per section within the VSMC layer were examined with an excitation wavelength of $450-500 \mathrm{~nm}$ and detection wavelength of 515-565 nm. As negative control, the slides were incubated in the absence of DNTT. For positive controls, the samples were first treated with DNase I for $10 \mathrm{~min}$ at $20^{\circ} \mathrm{C}$ to induce DNA strand breaks prior to labeling procedures and then incubated with $50 \mu \mathrm{L}$ of TUNEL reaction mixture. Two observers (K.C.A. and G.C.D.) who were unaware of the subjects' clinical profiles interpreted images independently and evaluated apoptotic indexes. Apoptotic index was calculated using the following formula: $100 \times$ (number of TUNEL-positive cell nuclei per field/total number of cell nuclei per field). The interobserver correlation was 2.26, whereas the intraclass correlation, obtained by a single observer who interpreted two images obtained at least 1 week apart from a single subject, was 0.94 .

\section{Immunofluorescent Analysis}

We applied ACTA2 (produced in mouse 1:100 dilution; SigmaAldrich Corp., St. Louis, Mo, USA) and anti-CASP3 antibody (produced in rabbit 1:100 dilution; Cell Signaling Technology, Inc., Danvers, MA, USA) after paraffin sections were incubated with the above blocking serum. Both antibodies were diluted in PBS, and incubations were applied at $37^{\circ} \mathrm{C}$ in a humidified chamber for 90 min. Cy5 goat anti-mouse IgG (Zymed Laboratories Inc., San Francisco, CA, USA) and Cy3 goat anti-mouse/anti-rabbit IgG 
Table 1 Characteristics of study subjects

\begin{tabular}{|c|c|c|c|c|}
\hline & Controls $(n=6)$ & TAA $(n=24)$ & $\operatorname{TAD}(n=20)$ & $P$ value* \\
\hline \multicolumn{5}{|l|}{ Age $(y r)$} \\
\hline Mean \pm SD & $37 \pm 7.2$ & $62.4 \pm 6.9$ & $54.4 \pm 10.6$ & \multirow[t]{2}{*}{$<0.0001$} \\
\hline Median (IQR) & $35.5(30.5 ; 44.25)$ & $62(56.25 ; 68)$ & $7.5(48.75 ; 62.75)$ & \\
\hline Male sex, $n(\%)$ & $5(83.3)$ & $17(70.8)$ & $14(70)$ & 0.8 \\
\hline History of hypertension, n (\%) & $0(0)$ & $17(70.8)$ & $15(75)$ & 0.002 \\
\hline History of smoking, n (\%) & $3(50)$ & $14(58.3)$ & $12(60)$ & 0.9 \\
\hline COPD, n (\%) & $0(0)$ & $7(29.2)$ & $6(30)$ & 0.3 \\
\hline Diabetes mellitus, $n(\%)$ & $0(0)$ & $9(37.5)$ & $6(30)$ & 0.20 \\
\hline \multicolumn{5}{|l|}{ Maximal aortic diameter (mm) } \\
\hline Mean \pm SD & $29.5 \pm 1.8$ & $58.6 \pm 2.7$ & $56.8 \pm 8.5$ & \multirow[t]{2}{*}{$<0.0001$} \\
\hline Median (IQR) & $29.5(27.75 ; 31.25)$ & $58(56.25 ; 60.75)$ & $58(53.5 ; 61.75)$ & \\
\hline Aortic regurgitant volume $\geq 60$ (mL/beat) & $0(0)$ & $14(58.3)$ & $9(45)$ & 0.04 \\
\hline \multicolumn{5}{|l|}{$\mathrm{SBP}(\mathrm{mmHg})$} \\
\hline Mean \pm SD & $111.6 \pm 11.6$ & $144.5 \pm 23.0$ & $147.2 \pm 17.5$ & \multirow[t]{2}{*}{0.004} \\
\hline Median (IQR) & $110(100 ; 122.5)$ & $150(130 ; 160)$ & $150(132.5 ; 160)$ & \\
\hline \multicolumn{5}{|l|}{ DBP (mmHg) } \\
\hline Mean \pm SD & $78.3 \pm 5.1$ & $86.8 \pm 11.4$ & $89 \pm 9.6$ & \multirow[t]{2}{*}{0.07} \\
\hline Median (IQR) & $80(70.75 ; 81.25)$ & $90(80 ; 95)$ & $90(81.25 ; 95)$ & \\
\hline \multicolumn{5}{|l|}{ Medications } \\
\hline Lipid-lowering medication, n (\%) & $0(0)$ & $13(54.2)$ & $9(45)$ & 0.057 \\
\hline ACE inhibitors/ARB, $n(\%)$ & $0(0)$ & $14(58.3)$ & $14(70)$ & 0.01 \\
\hline Beta-blockers, n (\%) & $0(0)$ & $11(45.8)$ & $15(75)$ & 0.004 \\
\hline Calcium antagonists, $\mathrm{n}(\%)$ & $0(0)$ & $8(33.3)$ & $4(20)$ & 0.2 \\
\hline
\end{tabular}

TAA, thoracic aortic aneurysm; TAD, thoracic aortic dissection; COPD, chronic obstructive pulmonary disease; SBP, systolic blood pressure; DBP, diastolic blood pressure; IQR, interquartile range; $A C E$, angiotensin converting enzyme; ARB, angiotensin receptor blocker. ${ }^{*} P$ values were calculated by pairwise comparisons after Kruskal-Wallis test for continuous variables and chi-square test for categorical variables.

(Zymed Laboratories Inc.) were used as secondary antibodies, respectively. Nuclei were stained with Sytox green $(1 \mu \mathrm{g} / \mathrm{mL})$ in $1: 1$ dilution of PBS/glycerol-mounting medium. Immunofluorescent labeling results were examined on a Zeiss LSM 510 Meta confocal laser scanning microscope (Carl Zeiss) using 488-nm argon ion, 543- and 633-nm helium neon lasers; 40× Plan-Apo oil and $100 \times$ Plan-Apo oil objectives. Single optical images were scanned with $1600 \times 1600$ frame size and $<1.2 \mu \mathrm{m}$ optical thicknesses. Length and area measurements and gray scale-based threshold (>50 was considered positive staining) tools in the LSM-510 software (version 3.2) were used to semiautomatically calculate the area. The detection parameters, such as laser intensity, amplifier offset and gain, and pinhole diameter, were fixed and kept at the same values for all specimens.

\section{Statistical Analysis}

Continuous variables (age, systolic blood pressure, diastolic blood pressure, aortic diameter, apoptosis index, and BAX/ BCL2 ratio) were given as mean \pm standard deviation or median (interquartile range: IQR) and were compared by using Kruskal-Wallis analysis. Pairwise comparisons were made by a method that has been previously defined [13] when KruskalWallis analysis revealed that a significant difference exists. We compared ordinal variables (BCL2, BAK1, BCL2L1 and BAX) using ordinal logistic regression with proportional odds model. In this analysis, odds of having higher categories of the ordi- nal variables were compared with that of having lower categories. Other categorical variables were compared by chisquare test. These analyses were performed using SPSS ${ }^{\circledR}$ Statistics 18.0 (SPSS Inc, Chicago, IL, USA). ACTA2 and CASP3 areas were compared by nonparametric analysis of covariance with adjusting for age. This analysis is performed in $\mathrm{R}$ software [14], and "sm.ancova" function is used under "sm" library [15]. We performed Benjamini-Hochberg procedure using "p.adjust" function to control the Type I error after pairwise comparisons [16]. A $P$ value of 0.05 was considered significant.

\section{Results}

\section{Clinical Characteristics and Assessment of Aortic} Disease in TAA and TAD Patients

Table 1 summarizes the clinical characteristics of the study cohort. Mean age for the aneurysm and dissection groups was significantly greater than control group. Range of maximum ascending aortic diameter in TAD and TAA patients assessed by computerized tomography, magnetic resonance imaging, or transesophageal echocardiography was between 36 and $70 \mathrm{~mm}$ $(56.8 \pm 8.5 \mathrm{~mm})$ and 54 and $65 \mathrm{~mm}(58.6 \pm 2.7 \mathrm{~mm})$, respectively. Aortic diameter in control patients was between 27 and $32 \mathrm{~mm}(29.5 \pm 1.8 \mathrm{~mm})$. All samples in TAA group were free from intraluminal thrombus. 
None of the groups demonstrated evidence of aortitis observed in H\&E staining. Aortic tunica media were significantly thinner in TAA $(421 \pm 29 \mu \mathrm{m}$; 95\% CI, 408-433) and TAD $(556 \pm 32 \mu \mathrm{m} ; 95 \%$ CI, 541-571) compared with that of control subjects $(1369 \pm 24 \mu \mathrm{m} ; 95 \%$ CI, 1343-1394; $P<0.001)$ as shown in Figure l(A). Moreover, VSMC area through ACTA2 within aortic tunica media was significantly reduced in TAA $\left(0.077 \pm 0.008 \mathrm{~mm}^{2}\right)$ and TAD $\left(0.074 \pm 0.007 \mathrm{~mm}^{2}\right)$ compared with that of control subjects $\left(0.185 \pm 0.011 \mathrm{~mm}^{2}\right)$ as shown in Figure 1(B). Thinning of the arterial wall, elastic fiber fragmentation along with disrupted architecture, and VSMC depletion were common in TAA and TAD groups according to confocal microscopic examination (Figure 1A,B). In the control group, VSMCs were arranged obliquely interconnected with the elastic laminae.

\section{Altered Expression of BCL2 Family of Proteins}

For a better understanding of molecular mechanisms of increased VSMC apoptosis in aneurysm degeneration and dissection, the expression pattern of different BCL2 family of proteins (BCL2, BCL2Ll, BAX, and BAK1) was investigated by immunohistochemistry in aortic sections (Figure 2). The intensity of these proteins was semiquantitatively scored [12] (Table 2). Furthermore, to assess the involvement of relevant protein expression, existence of positive immunoreactivity was evaluated. Proapoptotic BAX expression was significantly elevated in VSMCs of TAA patients, compared with that of controls $(\mathrm{OR}=20 ; P=0.02 ; 95 \% \mathrm{CI}$, 16-250). In contrast, antiapoptotic BCL2L1 expression was greater in controls compared with that of TAA group $(\mathrm{OR}=11.2$; $P=0.049 ; 95 \%$ CI $1.0-123.9)$. TAD group also showed a similar trend to TAA group with regards to BCL2L1 expression, but the results were not statistically significant when compared to controls $(\mathrm{OR}=7.6 ; P=0.06 ; 95 \% \mathrm{CI}, 0.9-50)$.

Furthermore, we investigated the ratio of BAX to BCL2 expression as previously described [17]. As the control group exhibited no immunoreactivity for proapoptotic BAX, BAX/BCL2 ratio was close to zero in the control group. BAX/BCL2 ratio was significantly increased in both TAA $(1.2 \pm 0.7, P<0.001)$ and TAD $(0.6 \pm 0.4, \quad P=0.05)$ groups relative to controls $(0.2 \pm 0.1$, $P<0.001)$.

\section{TAAs and TADs Exhibit Aortic VSMC Apoptosis}

All of the immunostaining and TUNEL staining were made in successive sections, which were $5 \mu \mathrm{m}$ apart, and the majority of cell type was VSMCs in media in all groups as confirmed by ACTA2 immunostaining (Figure 2). VSMC apoptosis was detected in 18 of 20 TAD sections (90\%) and 20 of 24 TAA sections $(83.3 \%)$, whereas negligible apoptosis was detected in control sections. Apoptotic index was significantly increased in both TAD group $(31.3 \pm 17.2, P<0.001)$ and TAA group $(21.1 \pm 12.7, P=0.001)$ relative to control aortas $(2.0 \pm 1.2)$ as shown in Figure 3. Furthermore, TAD group demonstrated higher apoptotic index compared with TAA group $(P=0.041)$.

To confirm that apoptosis is restricted to VSMCs, we further performed CASP3 and ACTA2 double immunolabeling. CASP3 immunoreactivity was colocalized with ACTA2 in medial layer of
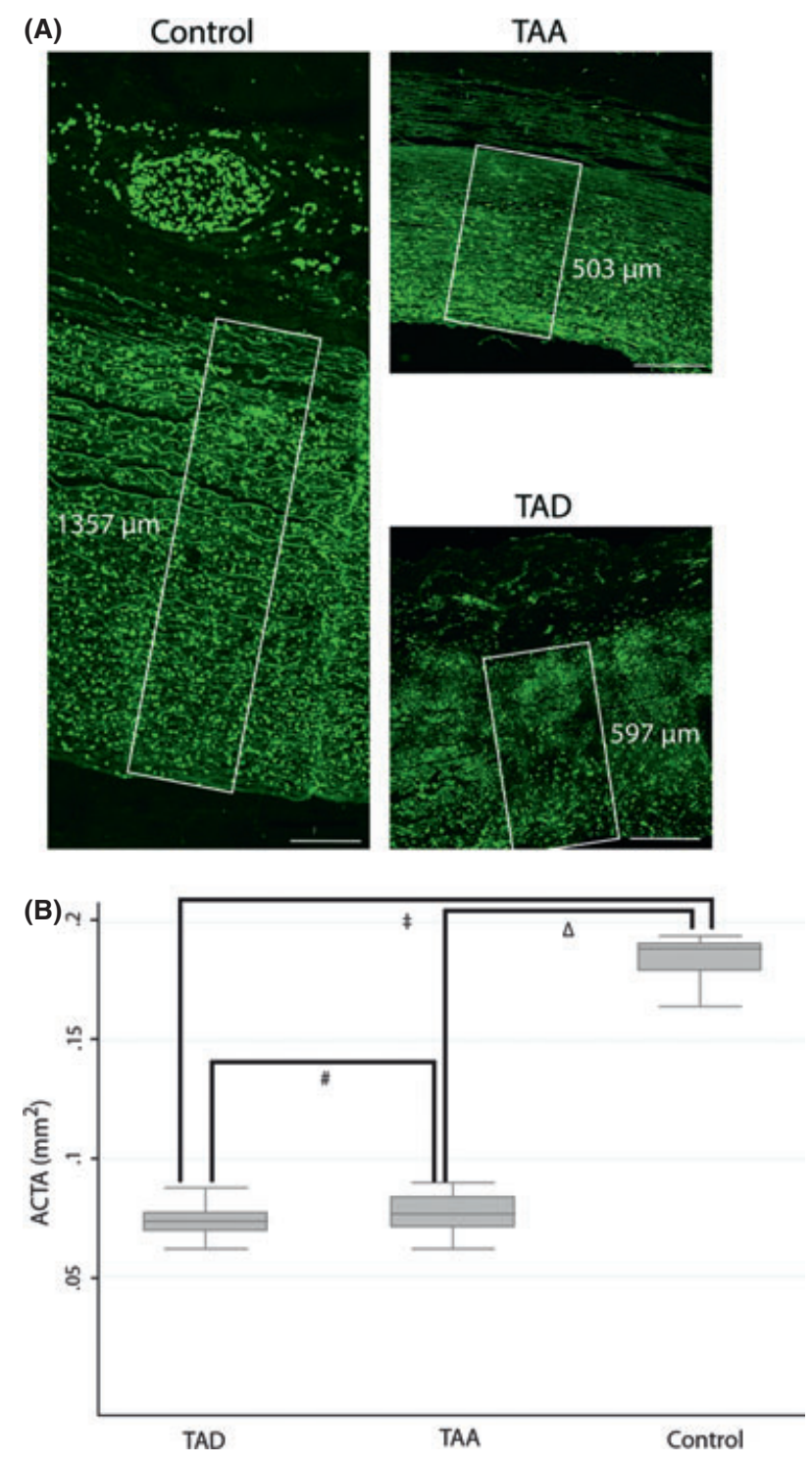

Figure 1 (A) Representative micrographs of tunica media immunostained for ACTA2 and demonstration of aortic wall thickness measurements in the aortas from control subjects and patients with thoracic aortic aneurysms (TAA) and type-A aortic dissections (TAD). Tunica media was thicker in a control subject (1357 $\mu \mathrm{m})$ compared with both TAA $(503 \mu \mathrm{m})$ and TAD $(597 \mu \mathrm{m})$ aortic samples. Additionally, a representative rectangular area covering the whole thickness of the tunica media was measured to calculate the total area of the positive ACTA2 signals as shown in (B). Box-whisker plots display calculate the total area of the positive ACTA2 signals. Central horizontal bars indicate median, upper and lower ends of boxes indicate 75th and 25th percentiles, and error bars indicate maximum and minimum values of total ACTA2 area ( $\left.{ }^{\#} P=0.211,{ }^{\ddagger} P=0.038,{ }^{\Delta} P=0.038\right)$. ACTA2 areas were compared by nonparametric analysis of covariance with adjusting for age. Scale bar is $200 \mu \mathrm{m}$.

TAA and TAD groups, whereas none or very little CASP3 staining was found in control group samples (Figure 4). CASP3 area measurement in aortic tunica media was significantly higher in 


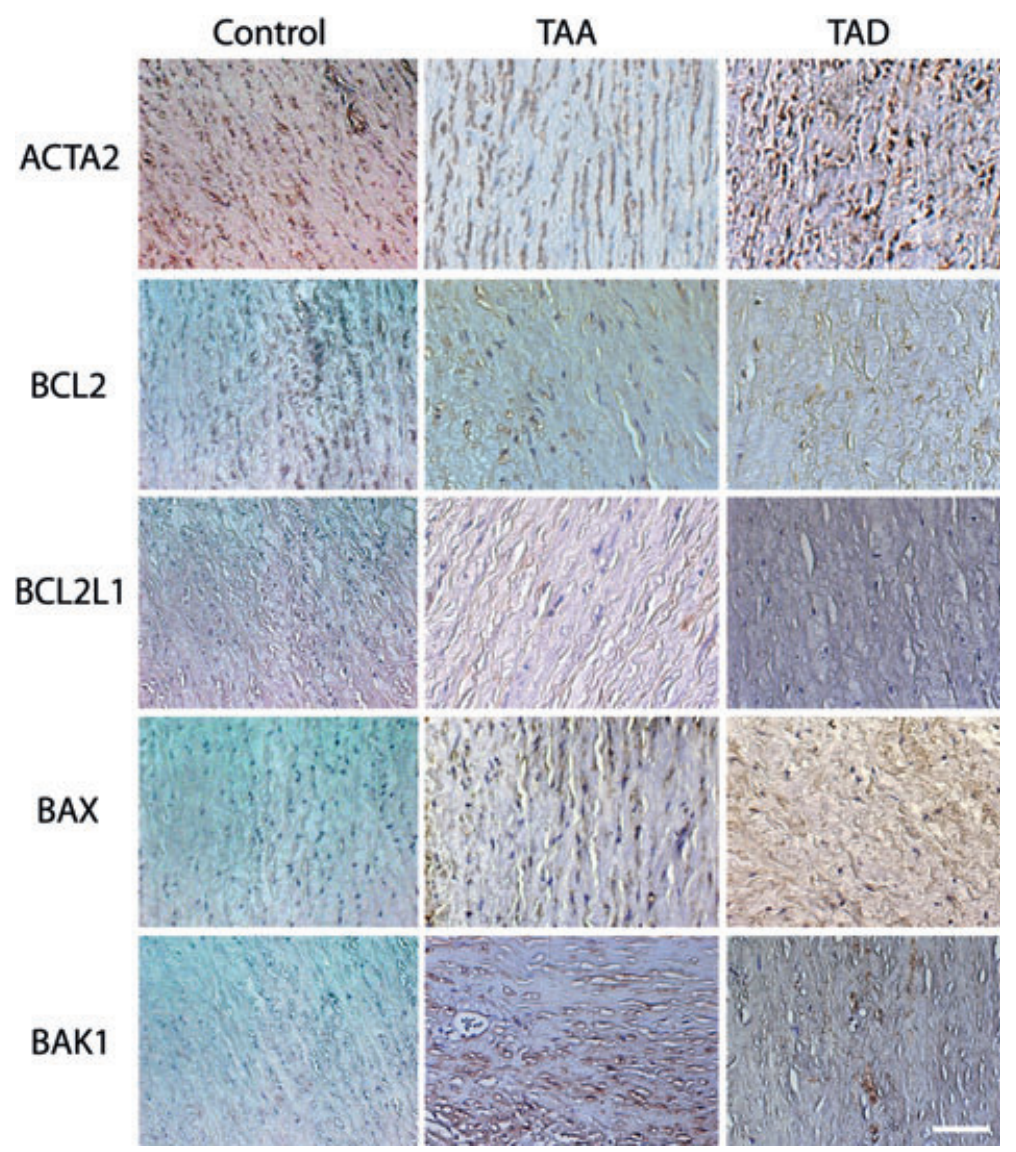

Figure 2 Representative micrographs for immunohistochemical stainings of serial aortic paraffin sections of the 27-mm ascending aorta of a 37-year-old man from the control group (left column); 67-mm ascending aortic aneurysm of a 66-year-old man from the thoracic aortic aneurysms (TAA) group (middle column); and 70-mm aortic aneurysm after acute type-A aortic dissection of a 64-year-old man from the type-A aortic dissections (TAD) group (right column) for ACTA2, antiapoptotic (BCL2, and BCL2L1), and proapoptotic (BAX, and BAK1) proteins. Elastic fiber fragmentation along with disrupted architecture and vascular smooth muscle cell (VSMC) depletion were common in TAA and TAD groups. Scale bar is $50 \mu \mathrm{m}$.

Table 2 The intensity of staining of BCL2 family proteins for control, TAA and TAD groups

\begin{tabular}{|c|c|c|c|c|c|}
\hline & Controls $(n=6)$ & TAA $(n=24)$ & $\operatorname{TAD}(n=20)$ & OR $(95 \% \mathrm{Cl})^{*}$ & $P$ value $^{\dagger}$ \\
\hline BCL2 score $(0 / 1 / 2 / 3 ; \%)^{*}$ & $0 / 33.3 / 33.3 / 33.3$ & $4.2 / 33.3 / 37.5 / 25$ & $10 / 45 / 25 / 20$ & TAD vs TAA: $0.64(0.19-2.12)$ & 0.5 \\
\hline Positive $(\%)^{\S}$ & 100 & 95.8 & 90 & $\begin{array}{l}\text { Control vs TAA: } 2.33(0.24-22.55) \\
\text { Control vs TAD: } 0.28(0.04-2.03)\end{array}$ & $\begin{array}{l}0.5 \\
0.2\end{array}$ \\
\hline $\begin{array}{l}\text { BCL2L1 score }(0 / 1 / 2 / 3 ; \%)^{\ddagger} \\
\text { Positive }(\%)^{\S}\end{array}$ & $\begin{array}{l}0 / 66.7 / 33.3 / 0 \\
100\end{array}$ & $\begin{array}{l}37.5 / 33.3 / 25 / 4.2 \\
62.5\end{array}$ & $\begin{array}{l}30 / 50 / 15 / 5 \\
70\end{array}$ & $\begin{array}{l}\text { TAD vs TAA: } 1.48(0.44-5.0) \\
\text { Control vs TAA: } 11.17(1.01-123.90) \\
\text { Control vs TAD: } 0.132(0.02-1.06)\end{array}$ & $\begin{array}{l}0.5 \\
0.05 \\
0.06\end{array}$ \\
\hline $\begin{array}{l}\text { BAX score }(0 / 1 / 2 / 3 ; \%)^{\ddagger} \\
\text { Positive }(\%)^{\S}\end{array}$ & $\begin{array}{l}50 / 50 / 0 / 0 \\
50\end{array}$ & $\begin{array}{l}8.3 / 20.8 / 33.3 / 37.5 \\
91.7\end{array}$ & $\begin{array}{l}15 / 60 / 20 / 5 \\
85\end{array}$ & $\begin{array}{l}\text { TAD vs TAA: } 0.18(0.05-0.67) \\
\text { Control vs TAA: } 0.05(0.004-0.63) \\
\text { Control vs TAD: } 3.49(0.44-27.87)\end{array}$ & $\begin{array}{l}0.01 \\
0.02 \\
0.3\end{array}$ \\
\hline $\begin{array}{l}\text { BAK1 score }(0 / 1 / 2 / 3 ; \%)^{\ddagger} \\
\text { Positive }(\%)^{\S}\end{array}$ & $\begin{array}{l}16.7 / 50 / 33.3 / 0 \\
83.3\end{array}$ & $\begin{array}{l}16.7 / 20.8 / 37.5 / 25 \\
83.3\end{array}$ & $\begin{array}{l}30 / 20 / 35 / 15 \\
70\end{array}$ & $\begin{array}{l}\text { TAD vs TAA: } 0.82(0.25-2.67) \\
\text { Control vs TAA: } 1.39(0.15-13.07) \\
\text { Control vs TAD: } 0.59(0.10-3.57)\end{array}$ & $\begin{array}{l}0.7 \\
0.8 \\
0.6\end{array}$ \\
\hline
\end{tabular}

TAA, thoracic aortic aneurysm; TAD, thoracic aortic dissection; OR, odds ratio; Cl, confidence interval; BCL2L1, BCL2-like 1; BAX, BCL2-associated X protein; BAK1, BCL2-antagonist/killer 1; BCL2, B-cell CLL/lymphoma 2. *Adjusted for age. ${ }^{\dagger} P$ values are calculated by using ordinal logistic regression with proportional odds model. "Immunoreactivity scored as 0 , not present; 1, light; 2 , moderate; 3 , high, respectively, and expressed as percentages of samples with the score. ${ }^{\S}$ Immunopositivity includes scores 1, 2, and 3 degree of scoring. 
(A)
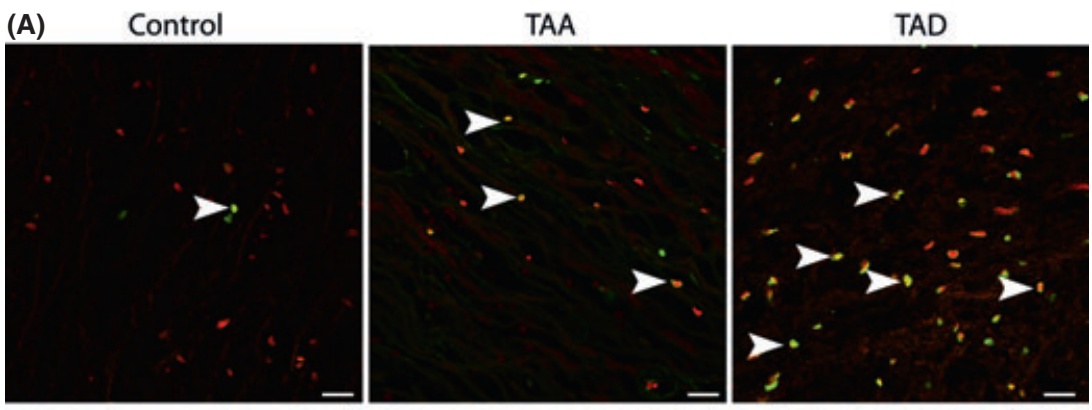

(B)
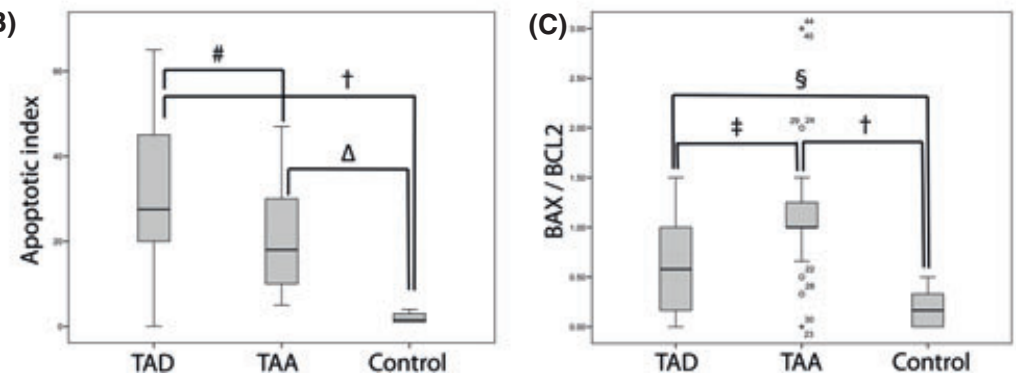

Figure 3 (A) Representative micrographs showing immunofluorescent TUNEL labeling within medial layer of serial aortic paraffin sections. Control, 27mm ascending aorta of a 37-year-old man from the control group; TAA, 67-mm ascending aortic aneurysm of a 66-year-old man; and TAA, 70-mm chronic aortic dissection and aneurysm of a 64-year-old man. Arrowheads indicate DNA fragmentation of TUNEL-positive apoptotic medial vascular smooth muscle cells (VSMCs). DNA fragmentation was detected in situ with a DNTT (terminal deoxynucleotidyl transferase)-mediated fluorescein-dUTP labeling kit (Roche Diagnostics, Mannheim, Germany) in the parallel sections according to manufacturer's instructions. To detect all nuclei, we used 7-aminoactinomycin D (7-AAD, Sigma; St Louis, MO). For each aortic sample, at least 10 grid fields per section within the vascular smooth muscle cell (VSMC) layer were examined with an excitation wavelength of 450-500 nm and detection wavelength of 515-565 nm. Scale bar is 20 $\mu$ m. (B) Box-whisker plots display apoptotic indexes. Central horizontal bars indicate median, upper and lower ends of boxes indicate 75 th and 25 th percentiles, and error bars indicate maximum and minimum values of apoptotic indexes. ( $\left.{ }^{\#} P=0.041,{ }^{\dagger} P<0.001,{ }^{\Delta} P=0.001\right)$ and $(\mathbf{C})$ box-whisker plots display BAX/BCL2 ratios. $\left({ }^{\dagger} P<0.001,{ }^{\S} P=0.05, \$ P=0.005\right)$. For each section, two independent observers counted TUNEL-positive cells, apoptotic indexes and BAX/BCL2 ratios; $P$ values were calculated by pairwise comparisons after Kruskal-Wallis test.

TAA $\left(0.030 \pm 0.009 \mathrm{~mm}^{2}\right)$ and TAD $\left(0.038 \pm 0.007 \mathrm{~mm}^{2}\right)$ compared with that in control subjects $\left(0.0007 \pm 0.00006 \mathrm{~mm}^{2}\right)$ as shown in Figure 4(C).

\section{Discussion}

This study has focused on VSMCs, which is a poorly explored area of the ascending aorta. We investigated whether medial VSMCs survive or resist to apoptosis during the progression of TAA and TAD. A number of previous studies have documented decreased VSMC density within the medial layer of AAA in contrast to atherosclerotic and normal human aortas and have shown evidence of VSMC apoptosis in AAAs [4-6]. VSMC apoptosis has also been documented in thoracic aortic disease including congenital aortic pathologies [18], bicuspid aortic valve [19], and thoracic aneurysms and dissections [7]. Our study confirms VSMC depletion and an increase in the amount of apoptotic cells by TUNEL staining in the media of both TAA and TAD patients. Furthermore, the novel contribution of the present study is the evaluation of the role of BCL2 family of proteins in apoptotic VSMC depletion in the human ascending aortas. Results presented in this study suggest that TAA and TAD have similar expression patterns for BCL2 family of proteins by potentiating proapoptotic members and minimizing antiapoptotic BCL2 proteins. In brief, apoptotic VSMC depletion in TAD and TAA is associated with increased BAX/BCL2 ratios.

Erdheim's cystic medial degeneration is an important histological sine qua non of TAAs and TADs, which is also detected, in congenital aortic disease such as Marfan syndrome, atherosclerosis, and aging [20]. mRNA expression studies revealed that TAAs were associated with pronounced increases in the expression of genes involved in regulating cell survival, proliferation, and programmed cell death including TNF- $\alpha$ and interleukin- $1 \beta$, which are potent inducers of apoptosis [21]. Wang et al. [22] had demonstrated increased expression of connective tissue growth factor and collagen deposition in the aortic wall of TAD patients. A number of causes may lead to the generation of apoptotic signals within the VSMCs, thereby impairing the maintenance of the arterial extracellular matrix and influencing the progression of aneurysms. With prolonged vessel wall stress and destructive proteases, the aorta undergoes a cascade of compensatory structural events.

Several factors may have contributed to the apoptotic signals and the expression pattern of BCL2 family of proteins in this study. Hypertension is the most consistent predisposing condition for TAAs and TADs. The reported incidence of hypertension in acute dissections is about $75 \%$ [23]. In the present study, the incidence of history of hypertension was $71 \%$ for TAA and $70 \%$ for TAD groups. Reactive oxygen species, nitric oxide, angiotensin 
(A)

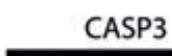

ACTA2

DNA

merge

Control
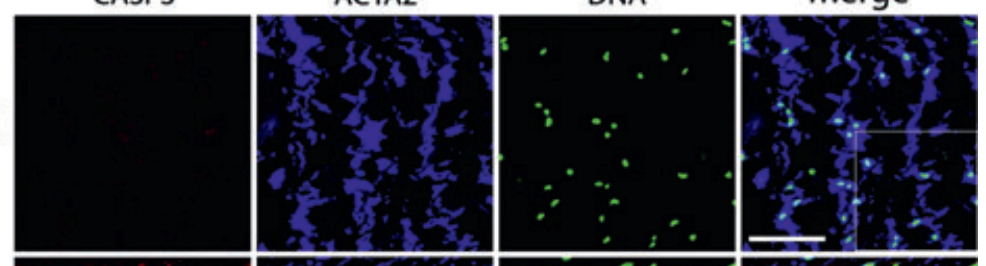

TAA
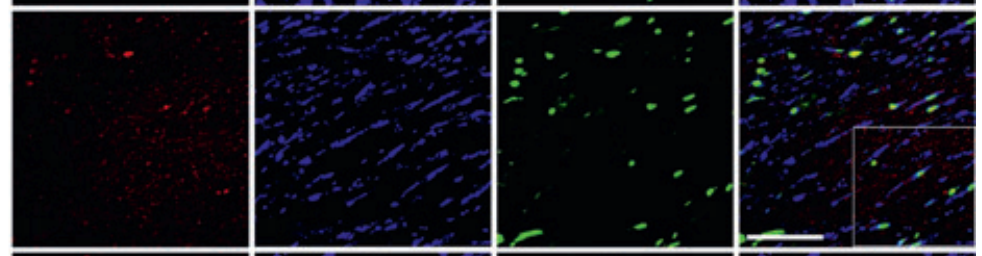

TAD
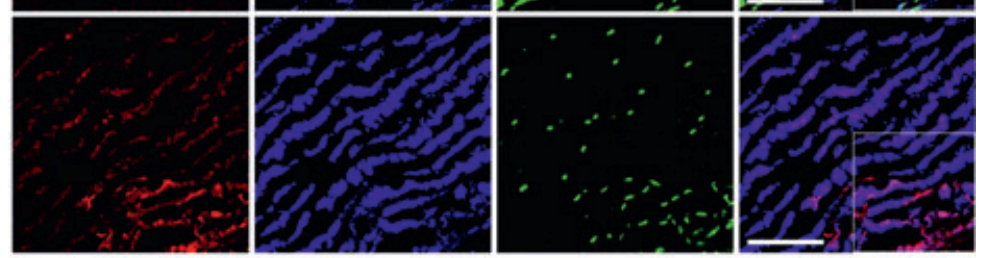

(B)

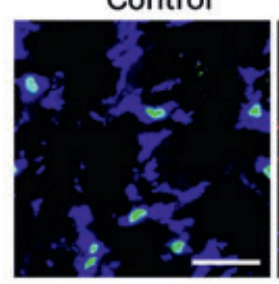

TAA

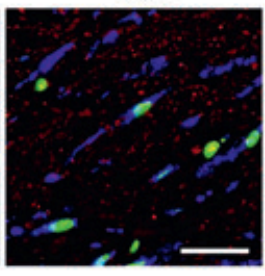

TAD

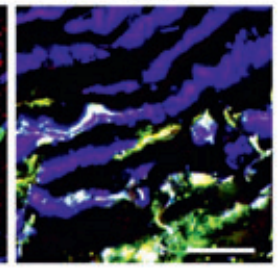

(C)

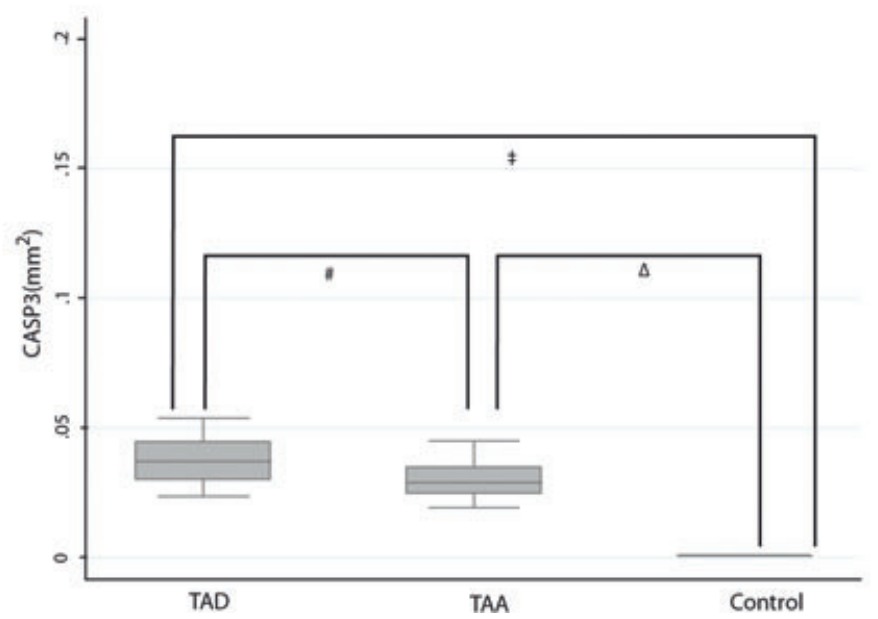

Figure 4 Caspase 3 (CASP3) activity in vascular smooth muscle cells (VSMCs). Confocal microscopic images of the aortas from control and patients with thoracic aortic aneurysm (TAA), type-A aortic dissections (TAD [CASP3, red signal; ACTA2, blue signal; nuclear labeling, green signal]). No or very little CASP3 activity is found in the control aortic samples, whereas disseminated CASP3 activity is detected within VSMCs of TAA and TAD patients shown in (A). Zoomed areas in (A) (insets) are illustrated in (B). CASP3 and ACTA2 signals were quantified, and the total size of the positive signals is given in (C). Box-whisker plots display the total calculated area of the positive CASP3 signals. Central horizontal bars indicate median, upper, and lower ends of boxes indicate 75th and 25th percentiles, and error bars indicate maximum and minimum values of total CASP3 area. $\left({ }^{\sharp} P=0.204,{ }^{\sharp} P=0.038,{ }^{\Delta} P=0.040\right)$. CASP3 areas were compared by nonparametric analysis of covariance with adjusting for age. Scale bars are $50 \mu \mathrm{m}(\mathbf{A})$ and $10 \mu \mathrm{m}$ (B).

type 2 receptors, and the endothelin system may play a role in the progression of hypertension and apoptosis of VSMCs [24]. Spontaneously, hypertensive [25] and angiotensin-infused rats [26] were found to have apoptosis detectable by DNA laddering, augmented in situ end-labeling of fragmented DNA, and/or BAX/BCL2 ratio. It has also been confirmed by Nagashima et al. [27] that angiotensin II type 2 receptor-mediated VSMC apoptosis had a pivotal role in patients with cystic medial degeneration. Detection of VSMC 
apoptosis in both TAA and TAD sections may also be related to other common predisposing factors such as flow and shear stress. For instance, recently Della et al. [28] demonstrated VSMC apoptosis with bicuspid aortic valve stenosis occurring prior to overt aortic dilation, mainly at the convexity, where wall stress is expectedly higher. As apoptosis is under control of complex cellular signalling pathways that are involved in responses to cellular stress signals and tissue injury, enhanced mechanical forces on medial VSMCs leading to stretch mediated reactions including programmed cell death via BAX upregulation [29]. However, we also determined an overall upregulation of antiapoptotic proteins BCL2 and BCL2L1 together with increased expression of BAX and BAK1.

According to our study design, the correlation between the stages of aortic wall expansion and initiation of BCL2 family of protein expression remains unclear. In this context, Bauriedel et al. [30] demonstrated the upregulation of BCL2 proteins after arterial injury. A certain degree of tendency for cell proliferation and survival at molecular level may also be accounted as the features of trophic response. Previously, Tang et al. [31] demonstrated decreased expression of matrix proteins as a result of increased matrix degradation but preserved VSMC density in patients with TAA. Our study supports the concept that VSMC apoptotic pathways may have a role in decreased expression of matrix proteins by documenting the relative cell depletion in the medial layer. Indeed, in agreement with our study, recent evidence supports the role of interleukin- $1 \beta$ and interferon- $\gamma$ in the formation of TAD and TAA through the upregulation of matrix metalloproteinase-9 and the apoptosis of human media cells [32].

Latest studies have shown that inflammatory cells, which infiltrate into vascular medial and adventitial layers, may cause apoptosis in AAA patients. Chemokine, cytokine and protease secretion from the infiltrated inflammatory cells are held as the probable cause of aneurysm pathogenesis, by leading resident vascular cells to apoptosis, which eventually cause a depletion in extracellular matrix proteins [33]. Immunohistocemical analyses of our TAA/TAD samples have shown absence or low-density $\mathrm{CD}^{+} \mathrm{T}$ cells, $\mathrm{CD} 63^{+}$macrophages, and tryptase ${ }^{+}$mast cells (data not shown). Thus, further research methods that focus on the detection of infiltrated inflammatory cells in samples from human ascending aorta and association of these cells with possible precursors of apoptosis would be a clinically relevant approach that could elucidate the mechanism of VSMC apoptosis in TAA and TAD patient groups.

It is also important to note that VSMC apoptosis might be associated with different expression patterns of BCL2 family of proteins in patients with TAA and TAD. However, the results presented cannot be generalized to all patients with aneurysms and dissections, because patients with inherited connective tissue disorders and any form of aortitis were excluded. In addition, microarray techniques revealed different profiles of altered gene expression and high degree of molecular heterogeneity in different locations of aortic aneurysms [21]. Thus, the findings cannot be extended to AAAs in which the embryonic origin of VSMCs is mesoderm [34]. The major limitations of this study were quantification of BCL2 family of proteins with methods such as Western blot or ELISA. However, our aim in this study was to demonstrate the expression patterns of these proteins within the aortic VSMCs.
Following homogenization to isolate BCL2 proteins, it would have been impossible to claim that these proteins are in fact originated from aortic VSMCs. Additionally, lack of immunocytochemistry and TUNEL assay prior to the event of TAD or in the early stages of aneurysms are lacking, which can be accomplished, in experimental models. Surgically removed relatively advanced stage aneurysmal specimens may not reflect earlier stages of aortic aneurysms or the state of impending rupture. Finally, three main questions still remain unanswered. Does the BCL2 family of protein expression start at the early stages of aneurysmal degeneration? Does the progression of TAD and development of aortic dissection change the expression profiles? And, how BCL2 family of protein expression is modulated in TAA and TAD patients?

\section{Conclusion}

The primary significance of the observations in the present study is that apoptotic VSMC depletion in TAD and TAA is associated with upregulation of death promoting and downregulation of survival promoting BCL2 family of proteins. BAX/BCL2 ratio, an apoptotic parameter that has been shown to be critical for the fate of the cell [35], was elevated in TAA and TAD samples compared with that of control aortas. Future research should consider targeting biological pathways including the BCL2 family of proteins in the context of apoptotic VSMC depletion in TAA or dissections. Our findings may lead to the conclusion that the apoptotic index can be used as a predictor of TAA, because we have shown that the apoptotic index in patients with TAA and TAD is higher compared with the control group. In the present study, it is established that the apoptotic cell death in VSMCs is involved in the development of the TAA and TAD. In the years to come, novel medical therapies that would target the proapoptotic cascade may benefit the TAA and TAD patients, by slowing down the progression of the pathology and even preventing the development of TAA and TAD.

\section{Acknowledgements}

We sincerely thank Duru Aras, Aynur Karadag, Bulent Kaya, Adnan Uysalel, and Kemalettin Ucanok for their support; Isinsu Kuzu and Nalan Akyurek for second opinion of immunohistochemical findings and helpful discussions; and the staff of Department of Pathology for their assistance; Mustafa Kilickap and Ozlem Ilk for their statistical review of the manuscript; Zeynep Bastuzel Eyileten and homograft laboratory for providing control aortic tissue samples.

\section{Funding Sources}

Ankara University School of Medicine Research Council and the Bilkent University Research Funds supported this work.

\section{Authors' Contributions}

SD, GCD, and ARA participated in the design of the study and drafted the manuscript. GCD and KCA carried out the immunohistochemical studies and the TUNEL assays. AC and DB performed the immunofluorescent analysis. CZ and AD participated 
in the data collection. SD, GCD, AC, CA, and ARA conceived the study, participated in its design and coordination, helped to draft the manuscript, and give final approval of the version to be published. SD, GCD, AC, AD, and ARA finalized the manuscript and responded to reviewers' comments. All authors read and approved the final manuscript.

\section{Conflict of Interest}

Drs Durdu and Cubukcuoglu Deniz contributed equally to this work. There is no undisclosed ethical problem or conflict of interest related to the submitted manuscript.

\section{References}

1. Kerr JF, Wyllie AH, Currie AR. Apoptosis: A basic biological phenomenon with wide-ranging implications in tissue kinetics. $\mathrm{Br} J$ Cancer 1972;26:239-257.

2. Clarke M, Bennett M, Littlewood T. Cell death in the cardiovascular system. Heart 2007;93: 659-664.

3. Sykes TC, Morris AG, Bradbury AW, Mosquera D. Apoptosis in vascular disease. Eur J Vasc Endovasc Surg 2001;22:389-395.

4. Lopez-Candales A, Holmes DR, Liao S, Scott MJ, Wickline SA, Thompson RW. Decreased vascular smooth muscle cell density in medial degeneration of human abdominal aortic aneurysms. Am J Pathol 1997; 150:9931007.

5. Henderson EL, Geng YJ, Sukhova GK, Whittemore AD, Knox J, Libby P. Death of smooth muscle cells and expression of mediators of apoptosis by t lymphocytes in human abdominal aortic aneurysms. Circulation 1999;99:96-104.

6. Rowe VL, Stevens SL, Reddick TT, Freeman MB, Donnell R, Carroll RC, Goldman MH. Vascular smooth muscle cell apoptosis in aneurysmal, occlusive, and normal human aortas. J Vasc Surg 2000;31:567-576.

7. He R, Guo DC, Estrera AL, et al. Characterization of the inflammatory and apoptotic cells in the aortas of patients with ascending thoracic aortic aneurysms and dissections. J Thorac Cardiovasc Surg 2006;131:671-678

8. Bhuiyan MS, Fukunaga K. Activation of htra2, a mitochondrial serine protease mediates apoptosis: Current knowledge on htra2 mediated myocardial ischemia/ reperfusion injury. Cardiovasc Ther 2008;26:224-232.

9. Martinou JC, Green DR. Breaking the mitochondrial barrier. Nat Rev Mol Cell Biol 2001;2:63-67.

10. Chipuk JE, Moldoveanu T, Llambi F, Parsons MJ, Green DR. The BCL-2 family reunion. Mol Cell 2010;37:299-310.

11. De Paepe A, Devereux RB, Dietz HC, Hennekam RC, Pyeritz RE. Revised diagnostic criteria for the marfan syndrome. Am J Med Genet 1996;62:417-426.

12. Akcali KC, Sahiner M, Sahiner T. The role of bcl-2 family of genes during kindling. Epilepsia 2005;46:217-223.

13. Conover WJ. Several independent samples. In: Conover WJ, editor. Practical Nonparametric Statistics, Wiley Series in Probability and
Statistics. New York: Jonh Wiley \& Sons, 1999;12.

14. R Core Development Team. R: A Language and Environment for Statistical Computing. Vienna: R Foundation for Statistical Computing, 2012. ISBN 3-900051-07-0, URL http://www. R-project.org/.

15. Bowman A, Azzalini A. sm: Smoothing methods for nonparametric regression and density estimation. R package version: 2.2-4.1. 2012. URL http://cran.r-project.org/web/packages/sm/ index.html

16. Benjamini Y, Hochberg Y. Controlling the false discovery rate: A practical and powerful approach to multiple testing. J R Stat Soc Ser B 1995;57:289-300.

17. Oltvai ZN, Milliman CL, Korsmeyer SJ. Bcl-2 heterodimerizes in vivo with a conserved homolog, bax, that accelerates programmed cell death. Cell 1993;74:609-619.

18. Bonderman D, Gharehbaghi-Schnell E, Wollenek G, Maurer G, Baumgartner H, Lang IM. Mechanisms underlying aortic dilatation in congenital aortic valve malformation. Circulation 1999;99:2138-2143.

19. Schmid FX, Bielenberg K, Schneider A, Haussler A, Keyser A, Birnbaum D. Ascending aortic aneurysm associated with bicuspid and tricuspid aortic valve: Involvement and clinical relevance of smooth muscle cell apoptosis and expression of cell death-initiating proteins. Eur J Cardiothorac Surg 2003;23:537-543.

20. Sakomura Y, Nagashima H, Aoka Y, et al. Expression of peroxisome proliferator-activated receptor-gamma in vascular smooth muscle cells is upregulated in cystic medial degeneration of annuloaortic ectasia in marfan syndrome. Circulation 2002;106:I259-I263.

21. Absi TS, Sundt TM 3rd, Tung WS, Moon M, Lee JK, Damiano RR Jr, Thompson RW. Altered patterns of gene expression distinguishing ascending aortic aneurysms from abdominal aortic aneurysms: Complementary DNA expression profiling in the molecular characterization of aortic disease. J Thorac Cardiovasc Surg 2003; 126:344-357.

22. Wang X, LeMaire SA, Chen L, et al. Increased collagen deposition and elevated expression of connective tissue growth factor in human thoracic aortic dissection. Circulation 2006; 1 14:I200-I205.

23. Hirst AE Jr, Johns VJ Jr, Kime SW Jr. Dissecting aneurysm of the aorta: A review of 505 cases. Medicine (Baltimore) 1958;37:217-279.

24. Intengan HD, Schiffrin EL. Vascular remodeling in hypertension: Roles of apoptosis, inflammation, and fibrosis. Hypertension 2001;38:581-587.
25. Sharifi AM, Schiffrin EL. Apoptosis in vasculature of spontaneously hypertensive rats: Effect of an angiotensin converting enzyme inhibitor and a calcium channel antagonist. Am J Hypertens 1998;11:1108-1116.

26. Diep QN, Li JS, Schiffrin EL. In vivo study of at (1) and at(2) angiotensin receptors in apoptosis in rat blood vessels. Hypertension 1999;34:617624.

27. Nagashima H, Sakomura Y, Aoka Y, et al. Angiotensin II type 2 receptor mediates vascular smooth muscle cell apoptosis in cystic medial degeneration associated with marfan's syndrome. Circulation 2001;104:I282-I287.

28. Della Corte A, Quarto C, Bancone C, et al. Spatiotemporal patterns of smooth muscle cell changes in ascending aortic dilatation with bicuspid and tricuspid aortic valve stenosis: Focus on cell-matrix signaling. $J$ Thorac Cardiovasc Surg 2008;135:8-18, el1-el2.

29. Ihling C, Szombathy T, Nampoothiri K, et al. Cystic medial degeneration of the aorta is associated with p53 accumulation, bax upregulation, apoptotic cell death, and cell proliferation. Heart 1999;82:286-293.

30. Bauriedel G, Jabs A, Skowasch D, et al. Dendritic cells in neointima formation after rat carotid balloon injury: Coordinated expression withanti-apoptotic bcl-2 and hsp47 in arterial repair. J Am Coll Cardiol 2003;42:930-938.

31. Tang PC, Coady MA, Lovoulos C, Dardik A, Aslan M, Elefteriades JA, Tellides G. Hyperplastic cellular remodeling of the media in ascending thoracic aortic aneurysms. Circulation 2005;112:1098-1105.

32. Zhang L, Liao MF, Tian L, et al. Overexpression of interleukin-lbeta and interferon-gamma in type i thoracic aortic dissections and ascending thoracic aortic aneurysms: Possible correlation with matrix metalloproteinase-9 expression and apoptosis of aortic media cells. Eur J Cardiothorac Surg 2011;40:17-22.

33. Xu JM, Shi GP. Emerging Role of Mast Cells and Macrophages in Cardiovascular and Metabolic Diseases. Endocr Rev 2012;33:71-108.

34. Lenk GM, Tromp G, Weinsheimer S, Gatalica Z, Berguer R, Kuivaniemi H. Whole genome expression profiling reveals a significant role for immune function in human abdominal aortic aneurysms. BMC Genomics 2007;8:237.

35. Festjens N, van Gurp M, van Loo G, Saelens X, Vandenabeele P. Bcl-2 family members as sentinels of cellular integrity and role of mitochondrial intermembrane space proteins in apoptotic cell death. Acta Haematol 2004;111: 7-27. 